\title{
Autophagy induced by baicalin involves downregulation of CD147 in SMMC-7721 cells in vitro
}

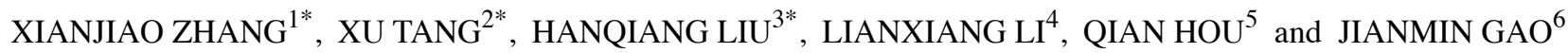 \\ ${ }^{1}$ School of Medicine, Xi'an Jiaotong University, Xi'an; ${ }^{2}$ Department of Pathology, Sichuan College of Traditional Chinese \\ Medicine, Mianyang; ${ }^{3}$ Department of Nutrition and Food Hygiene, The Fourth Military Medical University, Xi'an; \\ ${ }^{4}$ Department of Infection Control and Prevention, Shaanxi Provincial People's Hospital, Xi'an; ${ }^{5}$ Department \\ of Traditional Chinese Medicine, Xijing Hospital, The Fourth Military Medical University, Xi'an; \\ ${ }^{6}$ School of Public Policy and Administration, Xi'an Jiaotong University, Xi'an, P.R. China
}

Received October 10, 2011; Accepted November 29, 2011

DOI: $10.3892 / o r .2011 .1599$

\begin{abstract}
Baicalin has been demonstrated to exert anticancer effects mainly through induction of tumor cell apoptosis and cell cycle arrest. However, the precise mechanisms underlying its anticancer role remain to be elucidated. In the present study, we investigated whether autophagy was involved in the anticancer activity of baicalin in the human hepatocellular carcinoma (HCC) cell line SMMC-7721 and the possible molecular mechanisms. Our data showed that the viability of SMMC-7721 cells was significantly inhibited by baicalin in a dose- and time-dependent manner. Alongside apoptosis, autophagy was also induced by baicalin dose- and timedependently with the involvement of the autophagy-associated protein Beclin 1. Moreover, we demonstrated that cell death induced by baicalin was significantly inhibited by the apoptosis inhibitor z-DEVD-fmk or the autophagy inhibitor 3-MA, respectively. In addition, we found that $\mathrm{CD} 147$, a key molecule related both to apoptosis and autophagy, was markedly downregulated at the protein level in SMMC-7721 cells treated with baicalin. Collectively, this is the first study to suggest that baicalin induces autophagic cell death in SMMC-7721 cells, which involves the downregulation of CD147. Our study reveals a new mechanism for the anticancer effects of baicalin and puts forward a potential crucial role of CD147 in baicalininduced cancer cell death.
\end{abstract}

Correspondence to: Dr Xianjiao Zhang or Dr Jianmin Gao, School of Medicine, Xi'an Jiaotong University, 86 Box, 76 West Yanta Street, Xi'an 710061, P.R. China

E-mail: submissionzhang@163.com

E-mail: gaojm@mail.xjtu.edu.cn

*Contributed equally

Key words: baicalin, hepatocellular carcinoma cell, autophagy, apoptosis, CD147

\section{Introduction}

Hepatocellular carcinoma (HCC) is one of the most prevalent tumor types worldwide, especially in Asian countries, and both incidence and mortality rates of HCC have increased in recent years (1). Hepatic resection and transplantation are potential curative treatments for HCC, but only about $20 \%$ of the patients are suitable candidates (2). For the treatment of unresectable HCC, chemotherapy represents an important tool in the clinical therapy of HCC. However, severe cytotoxicity induced by most of the commonly used anticancer drugs on normal cells necessitates the screening and development of some novel therapeutic reagents with relatively low side effects (3). Recently, traditional Chinese medicines have been recognized as a new source of anticancer drugs and new adjuvant therapy to enhance the efficacy of chemotherapy and to reduce the cancer chemotherapy-related side effects (4). In this regard, Chinese traditional herbs have been of particular interest, due to their relatively low toxicity as concluded from their extensive clinical usage in the past. Isolation and screening of the active components from the herbs possessing anticancer potential appears to be a promising way of discovering novel therapeutic compounds.

The Chinese herb Scutellaria baicalensis (SB) is a member of the Lamiaceae or mint family and is known as Chinese skullcap (common name: Huang-Qin in China) and as Japanese Ogon. SB has been widely used in traditional Chinese medicine with multi-properties, such as antitumor, anti-inflammatory, anti-hypertensive, anti-cardiovascular, antibacterial and antiviral (4). Diverse phytoestrogen-like substances mainly including baicalin, baicalein and wogonin have been isolated from SB. Accumulating studies have demonstrated that baicalin possibly was the key bioactive ingredient mediating the anticancer effect of SB (4). Baicalin exerts strong suppressive effects on many types of cancer cells and has been documented to be a promising anticancer candidate (4-7). However, the precise mechanisms underlying its anticancer effects remain to be elucidated.

Autophagy is a highly conservative intracellular process for degrading long-lived proteins and cytoplasmic organelles that 
consists of several sequential steps: sequestration, transport to lysosomes, degradation and utilization of degradation products (8). It is characterized by the appearance of double- and multimembrane cytoplasmic vesicles that engulf cytoplasm and organelles, forming autophagosomes marked by microtubuleassociated protein light chain 3 (LC3) (9). Many key molecules are involved in this biological process, especially Beclin 1, a mammalian homolog of yeast Atg6/Vps30 and an essential regulator that promotes autophagosome formation through mediating the localization of other autophagy proteins on the pre-autophagosomal membrane (10). Autophagy plays a wide variety of roles in physiological and pathological processes, such as starvation adaptation (11), embryonic development (12), cell survival and death (13), and tumor suppression (14). Recent publications have reported two seemingly opposite functions of autophagy in tumor progression $(8,15)$. Based on the ability of autophagy to promote cell survival in response to metabolic stress, it has been suggested that autophagy may contribute to tumor development. On the other hand, autophagy also directly or indirectly induces autophagic cell death through excessive self-digestion and the activation of apoptosis and inhibits tumor progression (14). In this context, it is a novel anticancer strategy to induce autophagic cell death in cancer cells and this concept has been confirmed with several chemotherapy agents from traditional Chinese medicine such as berberine $(16,17)$ and arsenic trioxide $(18)$. However, it has never been investigated whether baicalin induces autophagy in cancer cells.

CD147, a glycosylated immunoglobulin superfamily transmembrane protein, is highly expressed in HCC cell lines and tumor tissues $(19,20)$. Several studies in vitro have suggested that CD147 inhibits tumor cell apoptosis (21) and anoikis (22), promotes invasion and metastasis $(19,23,24)$, enhances tumor angiogenesis (25), and conferrs chemoresistance to some drugs (22). These findings indicate that CD147 may serve as a key therapeutic target for HCC. Studies from our laboratory demonstrated that CD147 may play an important role in the inhibitory regulation of autophagy and autophagic cell death in HCC cells $(17,26)$. However, whether CD147 also plays a role in mediating the anticancer effects of baicalin remains unclear.

In the present study, we sought to assess the effects of baicalin on tumor cell growth, autophagy induction and the possible molecular mechanisms underlying baicalininduced cell death in SMMC-7721 cells in vitro. Our results demonstrate that baicalin induced both autophagic cell death and apoptosis with downregulation of CD147 expression in SMMC-7721 cells in vitro. To the best of our knowledge, this is the first study to investigate whether baicalin induces autophagy and the relationship between baicalin-induced cell death and CD147 expression.

\section{Materials and methods}

Materials. Baicalin was purchased from the China National Information Infrastructure for Reference Materials (Beijing, China) and dissolved in normal saline (NS) to $20 \mathrm{mg} / \mathrm{ml}$, $\mathrm{pH} 7.2$, then vortexed at room temperature for $10 \mathrm{~min}$. This solution was centrifuged at $5,000 \mathrm{rpm}$ for $10 \mathrm{~min}$ to remove any insoluble ingredients, then the supernatant was passed through a $0.22-\mu \mathrm{m}$ pore-size filter (Millipore, Bedford, MA) for sterilization and diluted in RPMI-1640 medium at a final concentration of $1 \mathrm{~mol} / \mathrm{l}(\mathrm{M})$ as a stock solution. The pEGFPLC3 plasmid was kindly provided by Professor Xingchun Gou (Institute of Basic Medicine, Xi'an Medical University, Xi'an, China). All reagents were obtained from common commercial sources.

Cell culture. SMMC-7721 cells were provided by the Institute of Cell and Biochemistry, Chinese Academy of Sciences (Shanghai, China) and cultured in RPMI-1640 medium supplemented with $10 \%$ heat-inactivated $\left(56^{\circ} \mathrm{C}\right)$ fetal bovine serum (FBS) and $100 \mathrm{U} / \mathrm{ml}$ penicillin, $100 \mu \mathrm{g} / \mathrm{ml}$ streptomycin at $37^{\circ} \mathrm{C}$ in a humidified atmosphere with $5 \% \mathrm{CO}_{2}$. To study baicalin-induced cell death, SMMC-7721 cells were cultured either with or without baicalin (treatment group or control group).

MTT assay. Cell viability was determined after treatment with various concentrations of baicalin and different durations of exposure to baicalin by the MTT assay as previously described (27). In brief, SMMC-7721 cells were seeded at a density of $5 \times 10^{3}$ cells/well in 96-well microplates in RPMI-1640 medium, and were cultured for $24 \mathrm{~h}$. Then, the cells were treated with various concentrations $(0,10,20,40,80$, and $160 \mu \mathrm{M})$ of baicalin for different times $(0,24,48$ or $72 \mathrm{~h})$. Subsequently, $10 \mu \mathrm{l}$ of $5 \mathrm{mg} / \mathrm{ml}$ MTT (Sigma Chemical Co., USA) was added to each well for an additional 4-h incubation and the resulting formazan crystals were dissolved in $100 \mu \mathrm{l}$ DMSO. Finally the optical density (OD) at $570 \mathrm{~nm}$ was measured using an automatic microplate reader (Immuno Mini NJ-2300; Inter Med, Tokyo, Japan). The percentage of cell viability inhibition was calculated according to the following formula: [(OD value of the control cells - OD value of the treated cells)/OD value of the control cells] x $100 \%$. The viability of the control cells, from the untreated cultures, was defined as $100 \%$.

Autophagy assay with LC3 dots. SMMC-7721 cells were transfected with pEGFP-LC3 for $24 \mathrm{~h}$ and then cultured with different concentration of baicalin $(0,40,80$ or $160 \mu \mathrm{M})$. Exogenous enhanced GFP (EGFP)-fused microtubuleassociated protein light chain 3 (EGFP-LC3) is a specific autophagy marker widely used in autophagy research (28). When autophagy is stimulated, the distribution pattern of GFP-LC3 changes from a diffuse cytoplasmic pattern to a punctate pattern that labels pre-autophagosomal and autophagosomal membranes (29). Autophagy was analyzed using an Olympus BX60 fluorescence microscope (Olympus, Tokyo, Japan). The percentage of cells with EGFP-LC3 punctate dots was determined as previously described (26). Briefly, a minimum of 100 cells from each sample was counted in three independent experiments. The percentage of cells showing EGFP-LC3 punctate dots was calculated by dividing the number of cells showing punctate dots by the number of cells counted.

Transmission electron microscope (TEM) analysis. A TEM analysis was performed as previously described (26). Briefly, after culture in the absence or presence of $160 \mu \mathrm{M}$ baicalin for $48 \mathrm{~h}$, SMMC-7721 cells were fixed with $3 \%$ glutaraldehyde 
in $0.2 \mathrm{M}$ phosphate buffer ( $\mathrm{pH} \mathrm{7.3)}$ for $4 \mathrm{~h}$ at $4^{\circ} \mathrm{C}$, then postfixed with $1 \%$ osmium tetroxide and $0.5 \%$ tannic acid for $1 \mathrm{~h}$ at $4^{\circ} \mathrm{C}$ followed by washing three times with $0.1 \mathrm{M}$ phosphate buffer (pH 7.3). Next, cells were dehydrated and embedded in Epon812 (Electron Microscopy Sciences, Fort Washington, PA, USA). Finally, sections were counterstained with uranyl acetate and lead citrate, and examined using a JEM-2000EX transmission Electron Microscope (Jeol Ltd., Tokyo, Japan).

Immunofluorescence staining. SMMC-7721 cells were stained for Beclin 1 (Abcam Co., USA) by immunofluorescence followin the protocol as detailed previously with the following modifications (30). Briefly, cells on coverslips were fixed in $4 \%$ paraformaldehyde for $15 \mathrm{~min}$ and permeabilized with $0.1 \%$ Triton X-100 for $10 \mathrm{~min}$, washed three times (PBS containing $0.01 \%$ Triton X-100 and 10\% FBS), followed by incubation with the primary antibody (anti-human Beclin 1) overnight at $4^{\circ} \mathrm{C}$, washed, and incubated with protein-blocking solution. Subsequently, cells were incubated for $1 \mathrm{~h}$ at room temperature with a secondary antibody (goat-anti mouse $\operatorname{IgG}$ ) that was conjugated to fluorescein isothiocyanate (FITC, green fluorescence) (Molecular Probes Inc., Eugene, Oregon, USA) and then washed. Finally, images were captured using an Olympus BX60 (Olympus Optical Co., Ltd., Tokyo, Japan) fluorescence microscope.

Western blot analysis. Beclin 1 and CD147 protein expression in SMMC-7721 cells was detected by Western blotting performed as previously described (25). Briefly, cells were washed twice with ice-cold PBS. Cell samples were lysed with RIPA buffer (Pierce Biotechnology, Inc., USA) for $45 \mathrm{~min}$ on ice and protein concentrations were measured using the BCA kit (Pierce Biotechnology, Inc.). Equal amounts of protein $(10 \mu \mathrm{g})$ were separated by $10 \%$ SDS-PAGE and electrophoretically transferred to polyvinylidene difluoride membranes (Millipore, Bedford, MA) using a mini trans-blot apparatus (Bio-Rad Laboratories). Membranes were blocked with PBS- $0.05 \%$ Tween-20 containing 5\% non-fat dry milk for $1 \mathrm{~h}$ and incubated with monoclonal mouse anti-human Beclin 1 and CD147 or tubulin antibody (Palo Alto, CA, USA) for $2 \mathrm{~h}$ at room temperature. Membranes were then washed three times with PBS- $0.05 \%$ Tween-20 and incubated with HRP-labeled goat anti-mouse antibody (Carlsbad, CA, USA) at a 1:10,000 dilution for $1 \mathrm{~h}$. Blots were developed using an enhanced chemiluminescence kit (Pierce Biotechnology, Inc.). Each experiment was repeated at least three times.

Trypan blue dye exclusion assay. Cell viability of SMMC-7721 cells was assessed by the trypan blue exclusion assay as previously described (31). Briefly, SMMC-7721 cells were pretreated with $0,40,80$ or $160 \mu \mathrm{M}$ baicalin for $48 \mathrm{~h}$ in the absence or presence of the autophagy specific inhibitor 3-MA $(100 \mu \mathrm{M})$ (Sigma Chemical Co.) and/or the caspase-3 specific inhibitor Zyloxy-Asp-Glu-Val-Asp fluoromethyl ketone (z-DEVD-fmk) $(100 \mathrm{nM})$ (Beyotime Institute of Biotechnology, China). Cells were washed with PBS, trypsinized, and collected by centrifugation, and then resuspended in $200 \mu \mathrm{l}$ PBS. After mixing with $200 \mu$ l of $0.8 \%$ trypan blue, the cells were counted using a hemocytometer. The number of dead cells with disrupted membranes (blue cells) per 200 cells was counted in tripli-

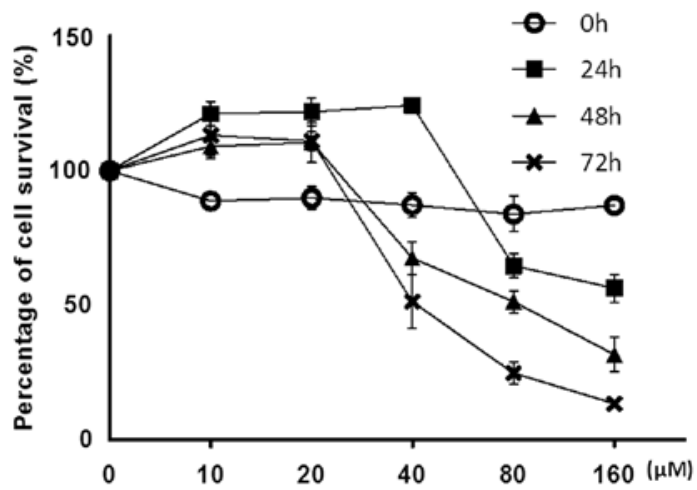

Figure 1. Baicalin inhibits SMMC-7721 cell proliferation in vitro. The viability of SMMC-7721 cells after baicalin treatment was analyzed using the MTT assay. The percentage of cell viability was calculated according to the following formula: [(OD value of the control cells - OD value of the treated cells)/OD value of the control cells] x $100 \%$. The viability of the control cells, from the untreated cultures, was defined as $100 \%$. Results are representative of three independent experiments. Error bars represent the standard deviation (SD). P-values were calculated using an unpaired Student's t-test.

cates. Cell death was expressed as the mean percentage of blue cells/total cells.

Statistical analysis. All statistical analyses were performed using the SPSS 16.0 statistical package for Microsoft Windows (SPSS, Chicago, IL). Statistical significance was determined using a Student's t-test. All tests in this study were two-sided and $\mathrm{P}<0.05$ was considered statistically significant.

\section{Results}

Antiproliferative effect of baicalin on SMMC-7721 cells. We first examined the effect of baicalin on the survival of SMMC-7721 cells using the MTT assay. Our results indicated that baicalin displayed strong inhibitory effect on the growth of SMMC-7721 cells in a dose and time-dependent manner (Fig. 1), and the $\mathrm{IC}_{50}$ value of baicalin was determined to be $40 \mu \mathrm{M}$ for $72 \mathrm{~h}$ or $80 \mu \mathrm{M}$ for $48 \mathrm{~h}$ (Fig. 1). Apparently, at a concentration as high as $160 \mu \mathrm{M}$, baicalin provoked the strongest inhibitory effect on SMMC-7721 cells, with morphological alterations characteristic of cell death observed under a light microscope (data not shown). However, at a low dose range (10 and $20 \mu \mathrm{M}$ ), baicalin failed to elicit a significant inhibitory effect on SMMC-7721 cells vitality. Thus, a baicalin concentration $\geq 40 \mu \mathrm{M}$ was used for subsequent experiments.

Autophagy induced by baicalin in SMMC-7721 cells. To determine whether baicalin could induce autophagy, the SMMC-7721 cells were transfected with a plasmid expressing an pEGFP-LC3 fusion protein and then exposed to different concentrations of baicalin $(40,80$ or $160 \mu \mathrm{M})$ for $48 \mathrm{~h}$. The formation of EGFP-LC3 punctate dots is a widely used marker for autophagy, which can be easily monitored by a fluorescence microscope (32). As expected, in normal condition without baicalin treatment, EGFP-LC3 fluorescence was largely diffused throughout the cytoplasm with few dots denoting basal autophagosome formation. However, the number of EGFP-LC3 dots rapidly increased within 24-h exposure to 
A
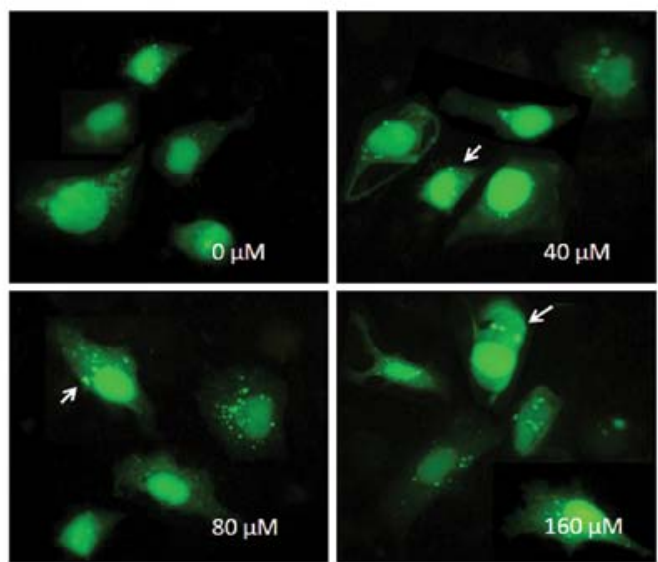

B

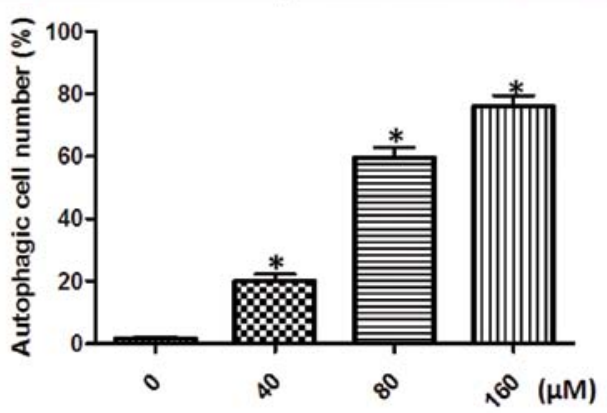

C

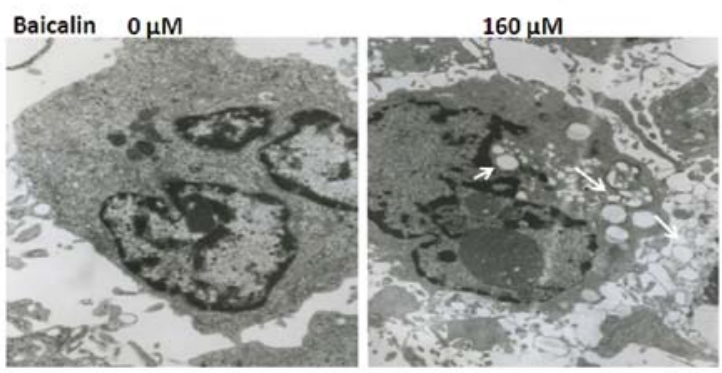

Figure 2. Baicalin induces autophagy in SMMC-7721 cells. SMMC-7721 cells were transfected with pEGFP-LC3, and then treated with the indicated concentrations of baicalin $(0,40,80$ and $160 \mu \mathrm{M})$ for $48 \mathrm{~h}$. Cells were viewed under a fluorescence microscope. Results shown are representative of three independent experiments. (A) Representative micrographs showing autophagosomes (white arrows). (B) Mean percentage of cells with LC3 punctate dots from triplicate samples in the different treatment groups. Error bars represent \pm SD. Statistical significance was determined using an unpaired Student's t-test. "P<0.01 vs. $0 \mu \mathrm{M}$. (C) SMMC-7721 cells were cultured with 0 or $160 \mu \mathrm{M}$ baicalin for $48 \mathrm{~h}$ before harvesting and analysis by transmission electron microscopy. The cells from the control group (without baicalin treatment) showed typical normal cancerous structures, with large and round nuclei, uniform chromatin density and clear nucleoli. However, the cells treated with $160 \mu \mathrm{M}$ baicalin for $48 \mathrm{~h}$ show signs of autophagy and apoptosis. White arrows indicate autophagic vesicles and white arrowheads indicate apoptosis.

baicalin (40, 80 or $160 \mu \mathrm{M})$, indicating that autophagy was induced. The percentage of autophagic cells with more than three GFP-LC3 puncta (GFP-LC3 positive) was $20.4 \%$ at $40 \mu \mathrm{M}, 61.2 \%$ at $80 \mu \mathrm{M}$, and $79.7 \%$ at $160 \mu \mathrm{M}$ baicalin, respectively, indicating that autophagy was induced by baicalin in a dose-dependent manner (Fig. 2A and B).

To further confirm the effect of baicalin on autophagy, we evaluated the level of baicalin-induced autophagy in SMMC-7721 cells using a TEM method, which is currently the standard method for monitoring autophagy. As shown in Fig. 2C, autophagic vacuoles (white arrows) were observed in
A
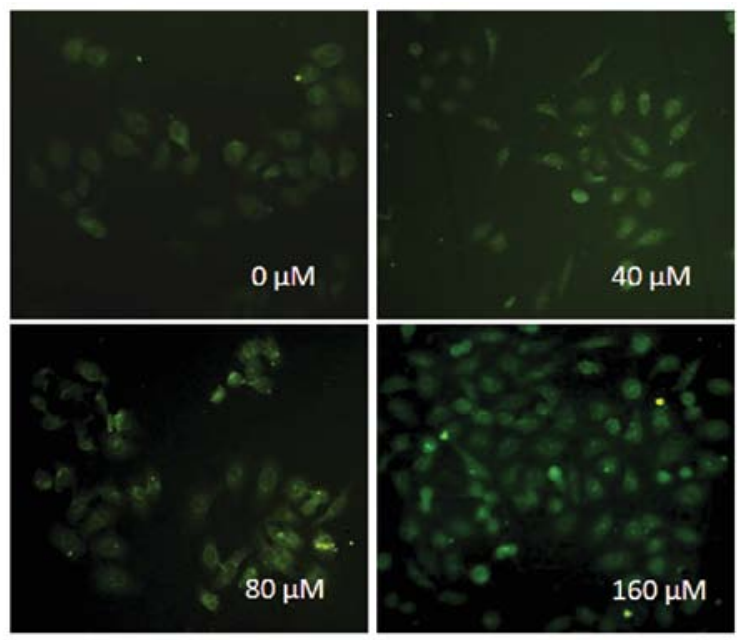

B

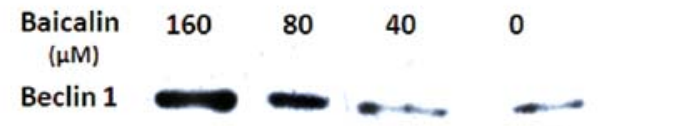

tubulin

Figure 3. Baicalin upregulates Beclin 1 protein levels in SMMC-7721 cells. (A) Immunofluorescence staining and (B) Western blot analyses for Beclin 1 protein levels in SMMC-7721 cells treated with baicalin $(0,40,80$ or $160 \mu \mathrm{M})$ for $48 \mathrm{~h}$.

SMMC-7721 cells treated with $160 \mu \mathrm{M}$ baicalin. The results suggest that a significantly higher level of autophagy occurred in SMMC-7721 cells treated with $160 \mu \mathrm{M}$ baicalin compared with that in the control ( $0 \mu \mathrm{M}$ baicalin). Moreover, we observed that cell nuclei had collapsed and disintegrated in cancer cells treated with baicalin (white arrowheads) (Fig. 2C), which indicated that baicalin also induced apoptosis and was in line with results of previous studies $(33,34)$. Taken together, all these results clearly demonstrated that baicalin induced autophagy as well as apoptosis in SMMC-7721 cells.

Baicalin upregulates autophagy-related protein Beclin 1 expression. To investigate the mechanisms by which baicalin induces autophagy, we detected the protein levels of Beclin 1, the key regulator of autophagy which has already been shown to be essential for the occurrence of autophagy (30). Following the treatment on SMMC-7721 cells with different concentrations of baicalin $(40,80$ or $160 \mu \mathrm{M})$ for $48 \mathrm{~h}$, we performed the immunofluorescence staining and Western blot analysis to evaluate the expression level of Beclin 1. Immunofluorescence staining showed that Beclin 1 abundance was remarkably higher in SMMC-7721 cells treated with baicalin compared to control in a dose-dependent manner (Fig. 3A). A similar result was obtained by Western blot analysis (Fig. 3B). These findings suggest that autophagy induced by baicalin in SMMC-7721 cells is, at least in part, Beclin 1-dependent.

Baicalin induces both autophagic cell death and apoptosis. Autophagy in cancer cells has been demonstrated to play a dual role in cell survival and cell death (15). To further determine the functional role of autophagy induced by baicalin in cancer 


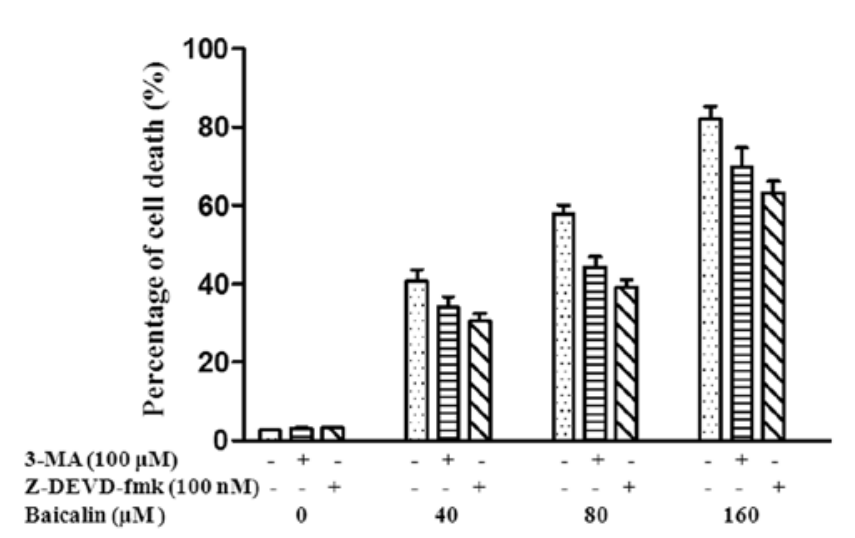

Figure 4. Baicalin induces autophagic cell death and apoptosis in SMMC7721 cells. SMMC-7721 cells were pretreated with various concentrations of baicalin $(0,40,80$ and $160 \mu \mathrm{M})$ in the absence or presence of 3-methyladenine (3-MA) and/or z-DEVD-fmk. The results show that both 3-MA and z-DEVD-fmk partly inhibited cell death. Data were presented as mean \pm SD of three separate experiments, each of which were performed in triplicate.

cell survival or death and the relationship between autophagy and apoptosis, we investigated baicalin-induced cell death in SMMC-7721 cells using a Trypan blue exclusion assay. 3-Methyladenine (3-MA), a known inhibitor of autophagy, was used to inhibit autophagy and prevent autophagic cell death induced alone (35). z-DEVD-fmk, a caspase-3-specific inhibitor of apoptosis, inhibits only apoptosis with no other types of cell death (36). SMMC-7721 cells were treated with 3-MA $(100 \mu \mathrm{M})$, z-DEVD-fmk (100 nM) or 3-MA $(0 \mu \mathrm{M})+$ z-DEVD-fmk (0 nM), and then cells were treated with 40, 80 and $160 \mu \mathrm{M}$ baicalin. The percentage of cell death was 33.9, 30.5 and $40.6 \%$, respectively in SMMC-7721 cells treated with $40 \mu \mathrm{M}$ baicalin. The percentage of cell death was $44.3,39.1$ and $57.7 \%$, respectively in SMMC-7721 cells treated with $80 \mu \mathrm{M}$ baicalin and 69.9, 63.3 and $82.1 \%$, respectively in SMMC-7721 cells treated with $160 \mu \mathrm{M}$ baicalin (Fig. 4). Collectively, our results demonstrate that cell death is inhibited by both 3-MA and z-DEVD-fmk, respectively, which further suggests that baicalin-induced cell death including both autophagic cell death and apoptosis.

Identification of cell death-related genes that regulate autophagy and apoptosis. Recent studies have uncovered significant interactions between autophagic and apoptotic signaling pathways $(37,38)$. It has been reported that autophagy and apoptosis may be linked to each other and occur simultaneously or sequentially in cancer cells (39). To better understand the relationship between autophagy and apoptosis in SMMC-7721 cells treated with baicalin, we investigated whether some known cell death genes related both to autophagy and apoptosis, were involved in baicalin-induced cell death. Previous studies have suggested that CD147 inhibited apoptosis (21) and starvation-induced autophagic cell death (26), and was related with cell cycle arrest in SMMC7721 cells (32). We thus investigated CD147 expression levels in SMMC-7721 cells treated with different concentrations of baicalin $(40,60,80,100,120$ or $160 \mu \mathrm{M})$. Western blotting results showed that CD147 expression level was significantly downregulated in SMMC-7721 cells treated with different

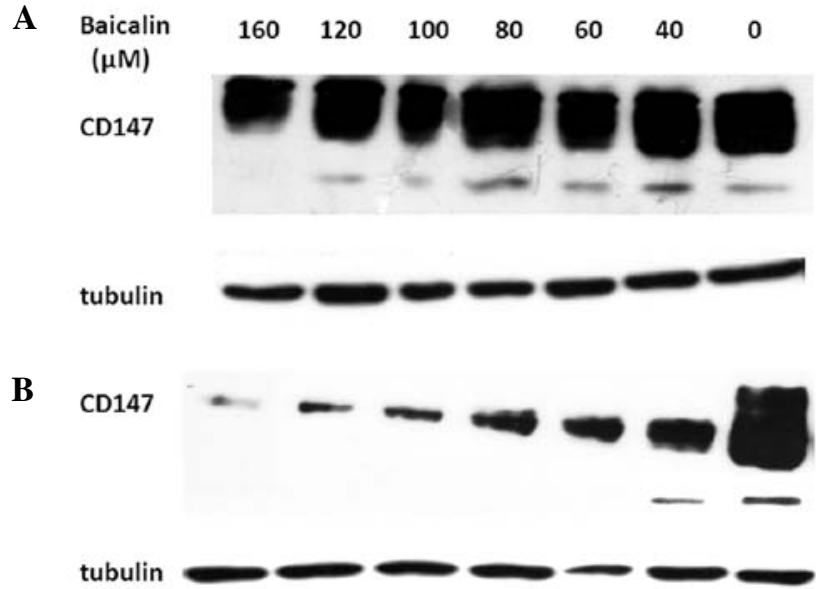

Figure 5. Baicalin downregulated CD147 expression. Western blot analysis for CD147 protein levels in SMMC-7721 cells treated with the different concentrations of baicalin $(0,40,60,80,100,120$ and $160 \mu \mathrm{M})$ for $(\mathrm{A}) 24 \mathrm{~h}$ or $48 \mathrm{~h}$ (B). Tubulin was used as a protein loading control in the Western blot assay.

concentrations of baicalin $(40,60,80,100,120$ and $160 \mu \mathrm{M})$ for $24 \mathrm{~h}$ (Fig. 5A) and $48 \mathrm{~h}$ (Fig. 5B) compared to the controls (cells without baicalin treatment) in a dose-dependent manner at protein levels. The data suggest that downregulation of CD147 expression may be involved in both baicalin-induced autophagy and apoptosis.

\section{Discussion}

Currently, chemotherapy using plant-derived anticancer drugs such as paclitaxel (40), vinorelbine (41), or vincristine (42) has been proven to be effective and safe in many clinical settings. It was shown that these products enhance cell growth inhibition, induce apoptosis or cell cycle arrest in several cancer cell lines and are highly effective and safe in clinical trials. Noticeably, about $75 \%$ of plant-derived drugs used today in the clinic originate from traditional medicines (43). Our previous result showed that berberine isolated from the Chinese herb Scutellaria baicalensis (SB) Georgi coule induced both apoptosis and autophagy in the HCC lines HepG2 and SMMC-7721 (17).

In the present study, we investigated the anticancer effects of baicalin, another ingredient isolated from SB, on HCC SMMC-7721 cells in vitro. Our results showed that baicalin exerted a significant antiproliferative effect on SMMC-7721 cells, in line with previous studies which demonstrated the antiproliferative effect of baicalin in other cancer cell lines (PC-3, DU145, LNCaP, MCF-7, HL-60, and NB4) $(33,34,44,45)$.

Our next attempt was to find out the underlying mechanisms of baicalin on SMMC-7721 cell proliferation inhibition. Previous studies indicated that baicalin induced apoptosis and cell cycle arrest in many types of cancers which contributed to cell growth inhibition $(33,34,44,45)$. However, whether baicalin induced other types of cell death such as autophagic cell death in cancer cells remains unknown. The present study is the first to provide compelling experimental evidence that baicalin induced autophagy in SMMC-7721 cells. This finding sheds new light on the pharmacological function of baicalin. Cancer is one of the first diseases genetically linked 
to autophagy malfunction $(8,46)$. The ATG gene Beclin 1 , as an important autophagy-associated signaling molecule, is mono-allelically deleted in $40-75 \%$ of human malignancies such as liver, lung, breast, ovarian, and prostate cancer (14). The restoration of Beclin 1 expression inhibits tumor cell proliferation and tumor growth (47). Moreover, upregulation of Beclin 1 expression mediates anticancer agent-induced autophagy (48). In the present study, we demonstrated that the expression of Beclin 1 was upregulated by baicalin in a dose-dependent fashion and positively associated with the level of autophagy, indicating that upregulation of Beclin 1 expression may be one of the major mechanisms of baicalininduced autophagy. The detailed mechanisms underlying the inter-relationships between autophagy, cell survival and cell death are largely unknown. Many studies showed that autophagy augmentation may be effective in preventing tumour formation and progression, whereas autophagy inhibition may be helpful in promoting tumour regression $(8,46)$. Currently, many studies have summarized the essential connections between defects in autophagy regulation or execution and cancer, and suggested that autophagy was a true tumour suppressor pathway (49). Several chemotherapy agents such as berberine $(16,17)$ and arsenic trioxide (18) have been demonstrated to induce autophagic cell death in cancer cells and thus exert anticancer activity. Therefore, in the present study we determined the functional role of autophagy induced by baicalin in SMMC-7721 cell survival or death. We further investigated whether baicalin could induce autophagic death in SMMC-7721 cells, defining a novel role of baicalin as an antitumor agent. Our results indicated that alongside apoptosis, baicalin also induced autophagic cell death in SMMC-7721 cells. These results suggest that induction of autophagy, as well as apoptosis, by baicalin may represent a novel mechanism by which baicalin inhibits tumor cell growth and modulates tumor progression.

Apoptosis (Type I) and autophagic cell death (Type II) are the two types of programmed cell death, whereas the boundary between Type I and II has never been completely clear and perhaps does not exist, and different types overlap. Autophagy and apoptosis have been reported to be linked to each other and occur simultaneously or sequentially in cancer cells (39). Recently, several laboratories have reported that molecules previously defined as intermediaries in the activation of apoptosis also function as intermediaries in the activation of autophagy $(37,50)$, suggesting these molecules may be involved in the modulation of both apoptosis and autophagy simultaneously. Targeting these molecules can be more effective than to target molecules modulating apoptosis or autophagy alone in cancer treatment. Thus, characterization of molecules that involve in the modulation of both apoptosis and autophagy has been actively pursued (51).

CD147 is the most commonly highly expressed gene in human cancers, mainly functions as a cellular adhesion molecule inducing the secretion of matrix metalloproteinases (MMPs) (19). Increased MMP expression was associated with tumor invasion and metastasis (19). Aside from stimulating MMPs production, CD147 also stimulates the production of vascular endothelial growth factor (VEGF) which serves as a major regulator of the angiogenic process in tumor formation (52). It has been reported that CD147 was associ- ated with the cell cycle, inhibited cancer cell apoptosis (21) and anoikis (22). Recent studies have further addressed that CD147 inhibited autophagy in SMMC-7721 cells with an involvement of Beclin 1 downregulation (26). These findings open the possibility that CD147 may be an important modulator involved in both apoptosis and autophagy. Given the fact observed in our present study that cell death induced by baicalin in SMMC-7721 cells included both apoptosis and autophagy. We evaluated the difference of the CD147 expression levels in SMMC-7721 cells treated with different concentrations of baicalin. As expected, the results indicated that CD147 expression levels were markedly downregulated by baicalin accompanied with the occurrence of apoptosis and autophagy. Based on these results, it is reasonable to hypothesize that baicalin-induced both apoptosis and autophagy in SMMC-7721 cells, at least in part, by downregulating CD147 expression and CD147 may serve as an important modulator of cross-talk between apoptosis and autophagy. However, further experiments are needed to verify the mechanisms by which baicalin regulates the expression of the CD147 and induce the cell death including apoptosis and autophagy.

In conclusion, our results show for the first time that baicalin induces autophagic cell death as well as apoptosis in SMMC-7721 cells, at least in part, through a pathway involving downregulation of CD147. These results suggest a novel mechanism underlying the pharmacological effects of baicalin and provide new insights into the function of CD147 during tumor progression.

\section{Acknowledgements}

The authors thank Professor Xingchun Gou (Institute of Basic Medicine, Xi'an Medical University, Xi'an, China) for valuable comments and providing pEGFP-LC3.

\section{References}

1. Jemal A, Siegel R, Xu J and Ward E: Cancer statistics, 2010. CA Cancer J Clin 60: 277-300, 2010.

2. Kassahun WT, Fangmann J, Harms J, Hauss J and Bartels M: Liver resection and transplantation in the management of hepatocellular carcinoma: a review. Exp Clin Transplant 4: 549-558, 2006.

3. Wang N, Tang LJ, Zhu GQ, et al: Apoptosis induced by baicalin involving up-regulation of P53 and bax in MCF-7 cells. J Asian Nat Prod Res 10: 1129-1135, 2008.

4. Li-Weber M: New therapeutic aspects of flavones: the anticancer properties of Scutellaria and its main active constituents Wogonin, Baicalein and Baicalin. Cancer Treat Rev 35: 57-68, 2009.

5. Ikemoto S, Sugimura K, Yoshida N, et al: Antitumor effects of Scutellariae radix and its components baicalein, baicalin, and wogonin on bladder cancer cell lines. Urology 55: 951-955, 2000.

6. Goh D, Lee YH and Ong ES: Inhibitory effects of a chemically standardized extract from Scutellaria barbata in human colon cancer cell lines, LoVo. J Agric Food Chem 53: 8197-8204, 2005.

7. Guo Y and Yao S: Effect of baicalin on invasion and metastasis of human hepatocellular line BEL-7402 in vitro. Acta Academiae Medicinae Militaris Tertiae 6: 33, 2006.

8. Mathew R, Karantza-Wadsworth V and White E: Role of autophagy in cancer. Nat Rev Cancer 7: 961-967, 2007.

9. Klionsky DJ and Emr SD: Autophagy as a regulated pathway of cellular degradation. Science 290: 1717-1721, 2000.

10. Cao Y and Klionsky DJ: Physiological functions of Atg6/ Beclin 1: a unique autophagy-related protein. Cell Res 17: 839-849, 2007.

11. Krustev LP: Cell autophagy of the liver in starvation and undernutrition. Bibl Nutr Dieta 23: 145-154, 1976. 
12. Qu X, Zou Z, Sun Q, et al: Autophagy gene-dependent clearance of apoptotic cells during embryonic development. Cell 128: 931-946, 2007.

13. Takagi H, Matsui Y and Sadoshima J: The role of autophagy in mediating cell survival and death during ischemia and reperfusion in the heart. Antioxid Redox Signal 9: 1373-1382, 2007.

14. Gozuacik D and Kimchi A: Autophagy as a cell death and tumor suppressor mechanism. Oncogene 23: 2891-2906, 2004.

15. Dalby KN, Tekedereli I, Lopez-Berestein G and Ozpolat B: Targeting the prodeath and prosurvival functions of autophagy as novel therapeutic strategies in cancer. Autophagy 6: 322-329, 2010.

16. Wang N, Feng Y, Zhu M, et al: Berberine induces autophagic cell death and mitochondrial apoptosis in liver cancer cells: the cellular mechanism. J Cell Biochem 111: 1426-1436, 2010.

17. Hou Q, Tang X, Liu H, et al: Berberine induces cell death in human hepatoma cells in vitro by downregulating CD147. Cancer Sci 102: 1287-1292, 2011.

18. Kanzawa T, Zhang L, Xiao L, Germano IM, Kondo Y and Kondo S: Arsenic trioxide induces autophagic cell death in malignant glioma cells by upregulation of mitochondrial cell death protein BNIP3. Oncogene 24: 980-991, 2004.

19. Xu J, Xu HY, Zhang Q, et al: HAb18G/CD147 functions in invasion and metastasis of hepatocellular carcinoma. Mol Cancer Res 5: 605-614, 2007.

20. Zhang Q, Zhou J, Ku XM, et al: Expression of CD147 as a significantly unfavorable prognostic factor in hepatocellular carcinoma. Eur J Cancer Prev 16: 196-202, 2007.

21. Kuang YH, Chen X, Su J, et al: RNA interference targeting the CD147 induces apoptosis of multi-drug resistant cancer cells related to XIAP depletion. Cancer Lett 276: 189-195, 2009.

22. Yang JM, O'Neill P, Jin W, et al: Extracellular matrix metalloproteinase inducer (CD147) confers resistance of breast cancer cells to Anoikis through inhibition of Bim. J Biol Chem 281: 9719-9727, 2006.

23. Dai J, Dou K, Wang C, et al: The interaction of HAb18G/CD147 with integrin $\alpha 6 \beta 1$ and its implications for the invasion potential of human hepatoma cells. BMC Cancer 9: 337, 2009.

24. Tang J, Wu YM, Zhao P, Yang XM, Jiang JL and Chen ZN: Overexpression of HAb18G/CD147 promotes invasion and metastasis via $\alpha 3 \beta 1$ integrin mediated FAK-paxillin and FAK-PI3K-Ca $2^{+}$pathways. Cell Mol Life Sci 65: 2933-2942, 2008.

25. Chen Y, Zhang H, Gou X, Horikawa Y, Xing J and Chen Z: Upregulation of HAb18G/CD147 in activated human umbilical vein endothelial cells enhances the angiogenesis. Cancer Lett 278: 113-121, 2009.

26. Gou X, Ru Q, Zhang H, et al: HAb18G/CD147 inhibits starvationinduced autophagy in human hepatoma cell SMMC7721 with an involvement of Beclin 1 down-regulation. Cancer Sci 100 837-843, 2009

27. Yuan SL, Wei YQ, Wang XJ, Xiao F, Li SF and Zhang J: Growth inhibition and apoptosis induction of tanshinone II-A on human hepatocellular carcinoma cells. World J Gastroenterol 10 : 2024-2028, 2004.

28. Kabeya Y, Mizushima N, Ueno T, et al: LC3, a mammalian homologue of yeast Apg8p, is localized in autophagosome membranes after processing. EMBO J 19: 5720-5728, 2000.

29. Qu X, Yu J, Bhagat G, et al: Promotion of tumorigenesis by heterozygous disruption of the beclin 1 autophagy gene. J Clin Invest 112: 1809-1820, 2003.

30. Liang XH, Yu J, Brown $\mathrm{K}$ and Levine B: Beclin 1 contains a leucine-rich nuclear export signal that is required for its autophagy and tumor suppressor function. Cancer Res 61: 3443-3449, 2001.

31. Strober W: Trypan blue exclusion test of cell viability. Curr Protoc Immunol Appendix 3: Appendix 3B, 2001.
32. Balgi AD, Fonseca BD, Donohue E, et al: Screen for chemical modulators of autophagy reveals novel therapeutic inhibitors of mTORC1 signaling. PLoS One 4: e7124, 2009.

33. Ikezoe T, Chen SS, Heber D, Taguchi H and Koeffler HP Baicalin is a major component of PC-SPES which inhibits the proliferation of human cancer cells via apoptosis and cell cycle arrest. Prostate 49: 285-292, 2001.

34. Chan FL, Choi HL, Chen ZY, Chan PSF and Huang Y: Induction of apoptosis in prostate cancer cell lines by a flavonoid, baicalin. Cancer Lett 160: 219-228, 2000.

35. Mizushima N, Yoshimori $\mathrm{T}$ and Levine B: Methods in mammalian autophagy research. Cell 140: 313-326, 2010.

36. Knoblach SM, Alroy DA, Nikolaeva M, Cernak I, Stoica BA and Faden AI: Caspase inhibitor z-DEVD-fmk attenuates calpain and necrotic cell death in vitro and after traumatic brain injury. $\mathrm{J}$ Cereb Blood Flow Metab 24: 1119-1132, 2004.

37. Pattingre S, Tassa A, Qu X, et al: Bcl-2 antiapoptotic proteins inhibit Beclin 1-dependent autophagy. Cell 122: 927-939, 2005.

38. Yousefi S, Perozzo R, Schmid I, et al: Calpain-mediated cleavage of Atg 5 switches autophagy to apoptosis. Nat Cell Biol 8: 1124-1132, 2006

39. Kondo Y, Kanzawa T, Sawaya R and Kondo S: Role of autophagy in cancer development and response to therapy. Nat Rev Cancer 5: 726-734, 2005

40. Gonzalez-Angulo AM, Walters RS, Carpenter JRJ, et al: Paclitaxel chemotherapy in a pregnant patient with bilateral breast cancer. Clin Breast Cancer 5: 317-319, 2004.

41. Romero A, Rabinovich MG, Vallejo CT, et al: Vinorelbine as first-line chemotherapy for metastatic breast carcinoma. J Clin Oncol 12: 336-341, 1994.

42. Holland JF, Scharlau C, Gailani S, et al: Vincristine treatment of advanced cancer: a cooperative study of 392 cases. Cancer Res 33: 1258-1264, 1973.

43. Newman DJ and Cragg GM: Natural products as sources of new drugs over the last 25 years. J Nat Prod 70: 461-477, 2007.

44. Franek KJ, Zengtong Z, Zhang WD and Chen WY: In vitro studies of baicalin alone or in combination with Salvia miltiorrhiza extract as a potential anti-cancer agent. Int J Oncol 26: 217-224, 2005

45. Xu XF, Cai BL, Guan SM, et al: Baicalin induces human mucoepidermoid carcinoma $\mathrm{Mc} 3$ cells apoptosis in vitro and in vivo. Invest New Drugs 29: 637-645, 2011.

46. Levine B and Kroemer G: Autophagy in the pathogenesis of disease. Cell 132: 27-42, 2008.

47. Liang XH, Jackson S, Seaman M, et al: Induction of autophagy and inhibition of tumorigenesis by beclin 1. Nature 402: 672-675, 1999.

48. Li DD, Wang LL, Deng R, et al: The pivotal role of c-Jun $\mathrm{NH}_{2}$-terminal kinase-mediated Beclin 1 expression during anticancer agents-induced autophagy in cancer cells. Oncogene: 28 : 886-898, 2008

49. Takahashi Y, Coppola D, Matsushita N, et al: Bif-1 interacts with Beclin 1 through UVRAG and regulates autophagy and tumorigenesis. Nat Cell Biol 9: 1142-1151, 2007.

50. Pattingre S and Levine B: Bcl-2 inhibition of autophagy: a new route to cancer? Cancer Res 66: 2885-2828, 2006.

51. Sun PH, Zhu LM, Qiao MM, et al: The XAF1 tumor suppressor induces autophagic cell death via upregulation of Beclin-1 and inhibition of Akt pathway. Cancer Lett 28: 170-180, 2011.

52. Tang Y, Nakada MT, Kesavan P, et al: Extracellular matrix metalloproteinase inducer stimulates tumor angiogenesis by elevating vascular endothelial cell growth factor and matrix metalloproteinases. Cancer Res 65: 3193-3199, 2005. 